\title{
Quantum coherence and criticality in irreversible work
}

\author{
Adalberto D. Varizi $\odot,{ }^{1,2}$ André P. Vieira $\odot,{ }^{1}$ Cecilia Cormick $\odot,{ }^{3}$ Raphael C. Drumond ${ }^{4}$ and Gabriel T. Landi ${ }^{1}$ \\ ${ }^{1}$ Instituto de Física da Universidade de São Paulo, 05314-970 São Paulo, Brazil \\ ${ }^{2}$ Departamento de Física, Instituto de Ciências Exatas, Universidade Federal de Minas Gerais, 30123-970, Belo Horizonte, \\ Minas Gerais, Brazil \\ ${ }^{3}$ Instituto de Física Enrique Gaviola, CONICET and Universidad Nacional de Córdoba, Ciudad Universitaria, \\ X5016LAE Córdoba, Argentina \\ ${ }^{4}$ Departamento de Matemática, Instituto de Ciências Exatas, Universidade Federal de Minas Gerais, 30123-970, \\ Belo Horizonte, Minas Gerais, Brazil
}

(Received 7 April 2020; accepted 3 August 2020; published 20 August 2020)

\begin{abstract}
The irreversible work during a driving protocol constitutes one of the most widely studied measures in nonequilibrium thermodynamics, as it constitutes a proxy for entropy production. In quantum systems, it has been shown that the irreversible work has an additional, genuinely quantum mechanical contribution, due to coherence produced by the driving protocol. The goal of this paper is to explore this contribution in systems that undergo a quantum phase transition. Substantial effort has been dedicated in recent years to understanding the role of quantum criticality in work protocols. However, practically nothing is known about how coherence contributes to it. To shed light on this issue, we study the entropy production in infinitesimal quenches of the one-dimensional $X Y$ model. We find that coherence plays a significant role in the entropy production and can even account for most of it in certain situations. Moreover, at low temperatures, the coherence presents a finite cusp at the critical point, whereas the entropy production diverges logarithmically. For high temperatures, however, the coherence presents a kink at the critical point indicating the quantum phase transition that occurs only at $T=0$. Alternatively, if the quench is performed in the anisotropy parameter, then we find that there are situations where all of the entropy produced is due to quantum coherences.
\end{abstract}

DOI: 10.1103/PhysRevResearch.2.033279

\section{INTRODUCTION}

Driving a system out of equilibrium is always accompanied by a finite production of entropy. The typical scenario is that shown in Fig. 1. A system with Hamiltonian $H(g)$, depending on an externally tunable parameter $g$, is initially prepared in thermal equilibrium at a temperature $T$, so that its density matrix is given by $\rho\left(g_{0}\right)=e^{-\beta H\left(g_{0}\right)} / Z\left(g_{0}\right)$, where $\beta=1 / T$ and $Z\left(g_{0}\right)$ is the partition function. At $t=0$ the system is driven out of equilibrium by changing $g$ according to some work protocol $g(t)$ that lasts for a total time $\tau$. If the dynamics can be considered unitary, then the state of the system after the drive will be

$$
\rho^{\prime}=U \rho\left(g_{0}\right) U^{\dagger},
$$

where $U=\mathcal{T} e^{-i \int_{0}^{\tau} H(g(t)) d t}$ is the time-evolution operator (with $\mathcal{T}$ standing for the time-ordering operator). This state is generally far from the corresponding equilibrium state $\rho\left(g_{\tau}\right)$; the difference between them can be quantified by the irreversible work [1-3],

$$
W_{\mathrm{irr}}=\langle W\rangle-\Delta F,
$$

Published by the American Physical Society under the terms of the Creative Commons Attribution 4.0 International license. Further distribution of this work must maintain attribution to the author(s) and the published article's title, journal citation, and DOI. where $\langle W\rangle=\operatorname{tr}\left\{H\left(g_{\tau}\right) \rho^{\prime}-H\left(g_{0}\right) \rho\left(g_{0}\right)\right\}$ is the average work performed in the process and $\Delta F=-T \ln Z\left(g_{\tau}\right) / Z\left(g_{0}\right)$ is the change in equilibrium free energy. Equation (2) can also be written solely in terms of information theoretic quantities (called the nonequilibrium lag), as [4-8]

$$
\Delta S_{\mathrm{irr}}=\beta W_{\mathrm{irr}}=S\left(\rho^{\prime} \| \rho\left(g_{\tau}\right)\right),
$$

where $S(\rho \| \sigma)=\operatorname{tr}(\rho \ln \rho-\rho \ln \sigma)$ is the quantum relative entropy. It thus measures the entropic distance between the final state $\rho^{\prime}$ and the associated equilibrium state $\rho\left(g_{\tau}\right)$ that the system does not tend to since the process is out of equilibrium (Fig. 1). Since $S\left(\rho^{\prime} \| \rho\left(g_{\tau}\right)\right) \geqslant 0$ by construction, this shows quite clearly why $\Delta S_{\text {irr }}$ or $W_{\text {irr }}$ can be used to quantify the nonequilibrium nature of the process $[1,3,9]$.

Strictly speaking, since the dynamics is unitary, no entropy is produced in the map Eq. (1). The nonequilibrium lag Eq. (3) is nonetheless a proxy for the entropy production. The reason is that, if after the protocol the system is once again coupled to a bath, it will relax from $\rho^{\prime}$ to $\rho\left(g_{\tau}\right)$, a process whose entropy production is precisely $\Delta S_{\text {irr }}$ in Eq. (3) [10-12]. For this reason, even though the process Eq. (1) is unitary, one commonly associates $\Delta S_{\text {irr }}$ with its entropy production [13].

This typical work-protocol scenario has been the subject of countless studies, both theoretical [1-7,14-86] as well as experimental [8,87-97] However, although Eq. (3) is formulated for quantum systems, many aspects of it are often classical. The issue of what are the genuinely quantum features of 


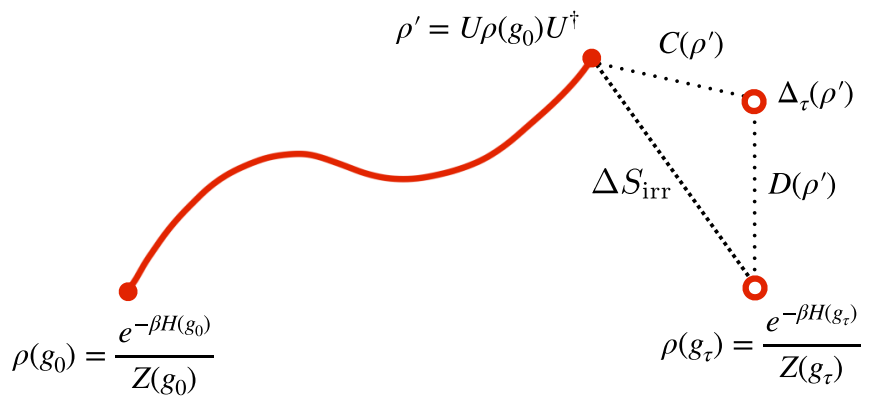

FIG. 1. Irreversible work and entropy production. A system with Hamiltonian $H(g)$ is initially prepared in equilibrium at a temperature $\beta=1 / T$, with $g=g_{0}$. The system is then pushed out of equilibrium by means of a work protocol $g(t)$, which lasts for a total duration $\tau$. This generates a unitary $U$ which pushes the system away from equilibrium to a state $\rho^{\prime}=U \rho\left(g_{0}\right) U^{\dagger}$. The nonequilibrium nature of the process can be quantified by the irreversible work $W_{\text {irr }}$ [Eq. (2)] or, what is equivalent, the entropy production/nonequilibrium lag $\Delta S_{\text {irr }}$ in Eq. (3). This quantity, however, can be split as in Eq. (4) into a contribution $D$ [Eq. (5)] related to changes in the population and a contribution $C$ related to quantum coherence [Eq. (6)].

such a process, despite still being the subject of debate, is ultimately related to the notion of quantum coherence. The thermodynamic processes involved in the map Eq. (1) highlight the energy basis as a preferred basis (in the sense of Ref. [98]). Coherence in the energy basis therefore represents the key feature distinguishing classical and quantum processes $[12,99]$. As the system is driven by the work protocol $g(t)$, the eigenbases of $H(g(t))$ at different times are not necessarily compatible, a feature which has no classical counterpart [63].

Several results have recently appeared, which highlight the nontrivial role of coherence in irreversible thermodynamics. For instance, Refs. [100,101] considered quasistatic drives and showed how the standard fluctuationdissipation theorem is modified to include a term related to $[H(g(t)), d H(g(t)) / d t]$, thus reflecting the basis incompatibility during the drive. In Ref. [12] some of us have shown that during relaxation to equilibrium, the presence of initial coherences contributes an additional term to the entropy production. A similar effect also occurs for unitary drives and the nonequilibrium lag, as shown in Ref. [61]. In this case, Eq. (3) may quite generally be decomposed as

$$
\Delta S_{\mathrm{irr}}=D\left(\rho^{\prime}\right)+C\left(\rho^{\prime}\right) .
$$

The first term quantifies the contribution from changes in the population of the system and reads

$$
D\left(\rho^{\prime}\right)=S\left(\Delta_{\tau}\left[\rho^{\prime}\right] \| \rho\left(g_{\tau}\right)\right),
$$

where $\Delta_{\tau}\left[\rho^{\prime}\right]$ is the completely dephased state, obtained from $\rho^{\prime}$ by eliminating its off-diagonal terms in the eigenbasis of $H\left(g_{\tau}\right)$. The second term in Eq. (4), however, is the relative entropy of coherence, given by

$$
C\left(\rho^{\prime}\right)=S\left(\rho^{\prime} \| \Delta_{\tau}\left[\rho^{\prime}\right]\right)=S_{v}\left(\Delta_{\tau}\left[\rho^{\prime}\right]\right)-S_{v}\left(\rho^{\prime}\right),
$$

where $S_{v}(\sigma)=-\operatorname{Tr}[\sigma \ln \sigma]$ is the von Neumann entropy. It therefore quantifies the difference between $\rho^{\prime}$ and the dephased state $\Delta_{\tau}\left[\rho^{\prime}\right]$. This term therefore measures the contribution to the nonequilibrium lag stemming solely from the quantum coherences generated by the driving protocol. Since both terms are individually nonnegative by construction, this shows how coherence increases the entropy produced in the process.

In this work we will be interested in the relative contributions of the two terms in Eq. (4) in the specific case of quantum critical systems undergoing infinitesimal quenches. That is, when the control parameter changes instantaneously from $g_{0} \rightarrow g_{\tau}=g_{0}+\delta g$, where $\delta g \ll g_{0}$. As shown in Refs. $[63,74,102,103]$, the nonequilibrium lag simplifies considerably in this case, since one removes the generally complicated dependence on the exact form of the work protocol $g_{\tau}$. Notwithstanding, the problem still retains several interesting features, particularly for quantum critical systems, as beautifully shown in Refs. [74,104]. This has led to a large number of studies on the critical properties of $\Delta S_{\text {irr }}$ in several models [82,105-112]. A proposal to measure it experimentally in ultracold atoms was also given in Ref. [113].

None of the studies above, however, dealt with the relative contribution from populations and coherences [Eq. (4)]. How relevant $C\left(\rho^{\prime}\right)$ is, therefore, remains unknown, even for the simplest critical models. It is the goal of this paper to fill in this gap and carry out a detailed study of the contribution from quantum coherence to the nonequilibrium lag in critical infinitesimal quenches. To accomplish this, we focus on the one-dimensional $X Y$ spin chain [114]. The advantage of this model is that by tuning the anisotropy parameter one may tune the relative contribution of $C\left(\rho^{\prime}\right)$ when going from the $X X$ to the transverse field Ising model. We show that for intermediate and high temperatures, both terms in Eq. (4) contribute similarly to $\Delta S_{\text {irr. }}$. At low temperatures, however, $C\left(\rho^{\prime}\right)$ becomes sub-dominant. And while $D\left(\rho^{\prime}\right)$ diverges logarithmically at the critical point $[82,104], C\left(\rho^{\prime}\right)$ presents a cusp.

\section{BASIC SETUP}

The Hamiltonian of the ferromagnetic $X Y$ model may be written as

$$
H(g, \gamma)=-\sum_{j=1}^{N}\left(\frac{1+\gamma}{2} \sigma_{j}^{x} \sigma_{j+1}^{x}+\frac{1-\gamma}{2} \sigma_{j}^{y} \sigma_{j+1}^{y}+g \sigma_{j}^{z}\right),
$$

where $\sigma_{j}^{a}(a=x, y, z)$ are Pauli spin operators, $N$ is the total number of spins, $\gamma \in[0,1]$ is the anisotropy parameter of the spin interaction and $g$ is the applied magnetic field. We assume $N$ even, with periodic boundary conditions. This model presents a paramagnetic phase when $|g|>1$ and a ferromagnetic phase for $|g|<1$, with critical points at $g^{*}=$ \pm 1 . Special cases occur when one makes $\gamma=0$, to get the $X X$ chain, and $\gamma=1$, to get the Ising model.

The Hamiltonian Eq. (7) is diagonalized by introducing the Jordan-Wigner transformation [115], that maps the spin chain onto an equivalent system of spinless fermions,

$$
\begin{aligned}
& \sigma_{j}^{x}=\left(\hat{c}_{j}^{\dagger}+\hat{c}_{j}\right) \prod_{i<j}\left(1-2 \hat{c}_{i}^{\dagger} \hat{c}_{i}\right), \\
& \sigma_{j}^{y}=\imath\left(\hat{c}_{j}^{\dagger}-\hat{c}_{j}\right) \prod_{i<j}\left(1-2 \hat{c}_{i}^{\dagger} \hat{c}_{i}\right), \quad \sigma_{j}^{z}=1-2 \hat{c}_{j}^{\dagger} \hat{c}_{j},
\end{aligned}
$$


where $\hat{c}_{j}^{\dagger}$ and $\hat{c}_{j}$ are canonical creation and annihilation fermionic operators. After this, one finds that the Hamiltonian Eq. (7) may be broken into two parts belonging to the orthogonal subspaces of positive and negative parity-i.e., subspaces of states with even or odd number of $c$-particles (or up spins), respectively. Each part can be independently diagonalized by a Fourier transform followed by a Bogoliubov transformation [116]. However, they differ only by boundary terms which become negligible in the thermodynamic limit $(N \rightarrow \infty)$. Hence, all calculations may therefore be performed considering only the positive parity subspace. We therefore consider here that, after diagonalization, we simply have

$$
H(g, \gamma)=\sum_{k \in K^{+}} \epsilon_{k}(g, \gamma)\left(2 \eta_{k}^{\dagger} \eta_{k}-1\right),
$$

where $K^{+}=\{k= \pm(2 n+1) \pi / N ; n=0,1,2, \ldots, N / 2-$ $1\}$. The dispersion relation $\epsilon_{k}(g, \gamma)$ is given by

$$
\epsilon_{k}(g, \gamma)=\sqrt{[g-\cos (k)]^{2}+\gamma^{2} \sin ^{2}(k)},
$$

and the canonical fermionic operators $\left\{\eta_{k}\right\}$, which depend on $g$ and $\gamma$, are given by

where

$$
\eta_{k}=\cos \left(\theta_{k} / 2\right) \hat{c}_{k}+\sin \left(\theta_{k} / 2\right) \hat{c}_{-k}^{\dagger},
$$

$$
\left(\sin \theta_{k}, \cos \theta_{k}\right)=\left(\frac{\gamma \sin (k)}{\epsilon_{k}(g, \gamma)}, \frac{g-\cos (k)}{\epsilon_{k}(g, \gamma)}\right),
$$

and

$$
\hat{c}_{j}=\frac{e^{-\imath \pi / 4}}{\sqrt{N}} \sum_{k \in K^{+}} \hat{c}_{k} e^{\imath k j} \text {. }
$$

For the special case $\gamma=0$, a Bogoliubov transformation is not necessary since the Hamiltonian $H_{\gamma=0}$ becomes diagonal after the Fourier transformation (10d), and is given by

$$
H_{\gamma=0}(g)=\sum_{k \in K^{+}}(g-\cos k)\left(2 \hat{c}_{k}^{\dagger} \hat{c}_{k}-1\right) .
$$

Our goal is to compute the entropic quantities appearing in Eqs. (5) and (6) for a quantum quench protocol. We initially consider the system to have an anisotropy parameter $\gamma_{0}$, transverse field $g_{0}$ and to be in equilibrium with a thermal reservoir at inverse temperature $\beta$. The initial state of the spin chain is therefore the thermal state $\rho(0)=e^{-\beta H(0)} / Z(0)$, with
$H(0)=H\left(g_{0}, \gamma_{0}\right)$ and partition function $Z(0)=\operatorname{Tr}\left[e^{-\beta H(0)}\right]$. Thus, can be further decomposed as

$$
\begin{aligned}
\rho(0) & =\bigotimes_{\substack{k \in K^{+} \\
k>0}} \rho_{ \pm k}^{0}, \\
\rho_{ \pm k}^{0} & =\frac{1}{Z_{k}^{2}(0)} \sum_{n_{ \pm k}=0}^{1} e^{2 \beta \epsilon_{k}^{0}\left(1-n_{k}-n_{-k}\right)}\left|n_{-k} n_{k}\right\rangle\left\langle n_{-k} n_{k}\right|,
\end{aligned}
$$

where $\left|n_{-k} n_{k}\right\rangle$ and $\epsilon_{k}^{0}=\epsilon_{k}\left(g_{0}, \gamma_{0}\right)$ are the eigenstates and eigenenergies of $H(0)$ and $Z_{k}(0)=2 \cosh \left(\beta \epsilon_{k}^{0}\right)$. The initial von Neumann entropy of this state is thus given by

$S_{v}(\rho(0))=\sum_{\substack{k \in K^{+} \\ k>0}} S_{v}\left(\rho_{ \pm k}^{0}\right)=2 \sum_{\substack{k \in K^{+} \\ k>0}}\left[\ln Z_{k}(0)-\beta \epsilon_{k}^{0} \tanh \left(\beta \epsilon_{k}^{0}\right)\right]$.

At $t=0$ the system is decoupled from the thermal reservoir and undergoes a sudden quench, where the field is instantaneously changed to $g_{\tau}$ and/or the anisotropy to $\gamma_{\tau}$. The Hamiltonian therefore changes from $H\left(g_{0}, \gamma_{0}\right)$ to $H\left(g_{\tau}, \gamma_{\tau}\right)$. Moreover, since we are considering a sudden quench, the state of the system does not change, so that $\rho^{\prime}=\rho(0)$. However, since in general $\left[H\left(g_{0}, \gamma_{0}\right), H\left(g_{\tau}, \gamma_{\tau}\right)\right] \neq 0$, the state $\rho^{\prime}$ will no longer be diagonal in the eigenbasis of $H\left(g_{\tau}, \gamma_{\tau}\right)$. To express $\rho^{\prime}$ in the new basis we first note that the post quench fermionic operators $\left\{\tilde{\eta}_{k}\right\}$ are related to the pre-quench operators $\left\{\eta_{k}\right\}$ according to [74]

$$
\tilde{\eta}_{k}=\eta_{k} \cos \left(\Delta_{k} / 2\right)+\eta_{-k}^{\dagger} \sin \left(\Delta_{k} / 2\right) \text {, }
$$

where $\Delta_{k}=\tilde{\theta}_{k}-\theta_{k}$ is the difference between the post- and pre-quench Bogoliubov angles Eq. (10c) and can be written as

$$
\sin \Delta_{k}=\frac{\sin k}{\epsilon_{k}^{\tau} \epsilon_{k}^{0}}\left[\gamma_{\tau}\left(g_{0}-\cos k\right)-\gamma_{0}\left(g_{\tau}-\cos k\right)\right],
$$

with $\epsilon_{k}^{\tau}=\epsilon_{k}\left(g_{\tau}, \gamma_{\tau}\right)$. As a consequence the pre- and postquench eigenstates will be related by

$$
\begin{aligned}
& \left|0_{-k} 0_{k}\right\rangle=\cos \left(\Delta_{k} / 2\right)\left|\tilde{0}_{-k} \tilde{0}_{k}\right\rangle-\sin \left(\Delta_{k} / 2\right)\left|\tilde{1}_{-k} \tilde{1}_{k}\right\rangle, \\
& \left|1_{-k} 1_{k}\right\rangle=\sin \left(\Delta_{k} / 2\right)\left|\tilde{0}_{-k} \tilde{0}_{k}\right\rangle+\cos \left(\Delta_{k} / 2\right)\left|\tilde{1}_{-k} \tilde{1}_{k}\right\rangle, \\
& \left|0_{-k} 1_{k}\right\rangle=\left|\tilde{0}_{-k} \tilde{1}_{k}\right\rangle, \quad\left|1_{-k} 0_{k}\right\rangle=\left|\tilde{1}_{-k} \tilde{0}_{k}\right\rangle .
\end{aligned}
$$

Using this in Eq. (12) we then find

$$
\begin{aligned}
\rho^{\prime}= & \bigotimes_{\substack{k \in K^{+} \\
k>0}} \tilde{\rho}_{ \pm k} \\
\tilde{\rho}_{ \pm k}= & \frac{1}{Z_{k}^{2}(0)}\left\{\left|\tilde{0}_{-k} \tilde{0}_{k}\right\rangle\left\langle\tilde{0}_{-k} \tilde{0}_{k}\left|\left[\cosh \left(2 \beta \epsilon_{k}^{0}\right)+\sinh \left(2 \beta \epsilon_{k}^{0}\right) \cos \Delta_{k}\right]+\right| \tilde{1}_{-k} \tilde{1}_{k}\right\rangle\left\langle\tilde{1}_{-k} \tilde{1}_{k}\right|\left[\cosh \left(2 \beta \epsilon_{k}^{0}\right)-\sinh \left(2 \beta \epsilon_{k}^{0}\right) \cos \Delta_{k}\right]\right. \\
& \left.+\left|\tilde{0}_{-k} \tilde{1}_{k}\right\rangle\left\langle\tilde{0}_{-k} \tilde{1}_{k}|+| \tilde{1}_{-k} \tilde{0}_{k}\right\rangle\left\langle\tilde{1}_{-k} \tilde{0}_{k}\right|-\left(\left|\tilde{0}_{-k} \tilde{0}_{k}\right\rangle\left\langle\tilde{1}_{-k} \tilde{1}_{k}|+| \tilde{1}_{-k} \tilde{1}_{k}\right\rangle\left\langle\tilde{0}_{-k} \tilde{0}_{k}\right|\right) \sinh \left(2 \beta \epsilon_{k}^{0}\right) \sin \left(\Delta_{k}\right)\right\} .
\end{aligned}
$$


We now use this to compute the relative entropy of coherence in Eq. (6). The state $\Delta_{\tau}\left[\rho^{\prime}\right]$ is obtained by taking only the diagonal entries of Eq. (17). As a consequence, one readily finds that

$$
\begin{aligned}
S_{v}\left(\Delta_{\tau}\left[\rho^{\prime}\right]\right)= & \sum_{\substack{k \in K^{+} \\
k>0}}\left\{2 \ln Z_{k}(0)-\frac{1}{2} \tanh \left(\beta \epsilon_{k}^{0}\right) \cos \left(\Delta_{k}\right) \ln \left[\frac{1+\tanh \left(2 \beta \epsilon_{k}^{0}\right) \cos \left(\Delta_{k}\right)}{1-\tanh \left(2 \beta \epsilon_{k}^{0}\right) \cos \left(\Delta_{k}\right)}\right]-\frac{\cosh \left(2 \beta \epsilon_{k}^{0}\right)}{4 \cosh ^{2}\left(\beta \epsilon_{k}^{0}\right)}\right. \\
& \left.\times \ln \left[1+\sinh ^{2}\left(2 \beta \epsilon_{k}^{0}\right) \sin ^{2}\left(\Delta_{k}\right)\right]\right\},
\end{aligned}
$$

Eq. (6) then follows from subtracting Eq. (13) from Eq. (18). We focus on the thermodynamic limit $(N \rightarrow \infty)$, where all $k$-sums may be converted into integrals. Moreover, we study the relative entropy of coherence per particle as $C\left(\rho^{\prime}\right)=C\left(\rho^{\prime}\right) / N$. In the limit $N \rightarrow \infty$ one then finds

$$
\begin{aligned}
C\left(\rho^{\prime}\right)= & \int_{0}^{\pi} \frac{d k}{2 \pi}\left\{\frac{1}{2} \tanh \left(\beta \epsilon_{k}^{0}\right)\left[\ln \left[\frac{1+\tanh \left(2 \beta \epsilon_{k}^{0}\right)}{1-\tanh \left(2 \beta \epsilon_{k}^{0}\right)}\right]-\cos \left(\Delta_{k}\right) \ln \left[\frac{1+\tanh \left(2 \beta \epsilon_{k}^{0}\right) \cos \left(\Delta_{k}\right)}{1-\tanh \left(2 \beta \epsilon_{k}^{0}\right) \cos \left(\Delta_{k}\right)}\right]\right]-\frac{\cosh \left(2 \beta \epsilon_{k}^{0}\right)}{4 \cosh ^{2}\left(\beta \epsilon_{k}^{0}\right)}\right. \\
& \left.\times \ln \left[1+\sinh ^{2}\left(2 \beta \epsilon_{k}^{0}\right) \sin ^{2}\left(\Delta_{k}\right)\right]\right\} .
\end{aligned}
$$

A similar calculation was done for the nonequilibrium lag $\Delta \mathcal{S}_{\text {irr }}=\Delta S_{\text {irr }} / N$ in Ref. [82], which found

$$
\Delta \mathcal{S}_{\mathrm{irr}}=\int_{0}^{\pi} \frac{d k}{2 \pi} 2\left\{\ln \left[\frac{\cosh \left(\beta \epsilon_{k}^{\tau}\right)}{\cosh \left(\beta \epsilon_{k}^{0}\right)}\right]+\beta\left(\epsilon_{k}^{0}-\epsilon_{k}^{\tau} \cos \left(\Delta_{k}\right)\right) \tanh \left(\beta \epsilon_{k}^{0}\right)\right\} .
$$

This result for the nonequilibrium lag can also be derived directly from the general expression of the characteristic function of work for arbitrary protocols in the $X Y$ model computed in Ref. [85]. From Eqs. (19) and (20), $D\left(\rho^{\prime}\right)$ in Eq. (5) can be readily computed using Eq. (4). Focusing again on the contribution per particle, $\mathcal{D}\left(\rho^{\prime}\right)=D\left(\rho^{\prime}\right) / N$, one then finds

$$
\begin{aligned}
\mathcal{D}\left(\rho^{\prime}\right)= & \int_{0}^{\pi} \frac{d k}{2 \pi}\left\{2 \ln \left[\frac{\cosh \left(\beta \epsilon_{k}^{\tau}\right)}{\cosh \left(\beta \epsilon_{k}^{0}\right)}\right]-\frac{1}{2} \tanh \left(\beta \epsilon_{k}^{0}\right) \cos \left(\Delta_{k}\right)\left[\ln \left[\frac{1+\tanh \left(2 \beta \epsilon_{k}^{\tau}\right)}{1-\tanh \left(2 \beta \epsilon_{k}^{\tau}\right)}\right]-\ln \left[\frac{1+\tanh \left(2 \beta \epsilon_{k}^{0}\right) \cos \left(\Delta_{k}\right)}{1-\tanh \left(2 \beta \epsilon_{k}^{0}\right) \cos \left(\Delta_{k}\right)}\right]\right]\right. \\
& \left.+\frac{\cosh \left(2 \beta \epsilon_{k}^{0}\right)}{4 \cosh ^{2}\left(\beta \epsilon_{k}^{0}\right)} \ln \left[1+\sinh ^{2}\left(2 \beta \epsilon_{k}^{0}\right) \sin ^{2}\left(\Delta_{k}\right)\right]\right\} .
\end{aligned}
$$

As a sanity check, in the case of an $X X$ chain $\left(\gamma_{0}=\gamma_{\tau}=0\right)$ the quench does not affect the eigenbasis so $\Delta_{k}=0$. Hence, $C\left(\rho^{\prime}\right)=0$, and all contributions to the nonequilibrium lag stems from the changes in populations.

\section{HIGH- AND LOW-TEMPERATURE LIMITS}

Since these results are somewhat complicated, we now proceed to separately analyze some limiting cases. As a consistency check, in all numerical analyses presented in this section, the integrals in Eqs. (19)-(21) were compared with exact numerics; i.e., obtained from discrete summations over the set $K^{+}$[cf. Eq. (18)] for sufficiently large $N$.

\section{A. High-temperature limit}

For small $\beta$ (high temperatures), the expressions for $C\left(\rho^{\prime}\right)$, $\mathcal{D}\left(\rho^{\prime}\right)$ and $\Delta \mathcal{S}_{\text {irr }}$ simplify dramatically to

$$
\begin{aligned}
& \mathcal{C}\left(\rho^{\prime}\right)=\beta^{2} \int_{0}^{\pi} \frac{d k}{2 \pi}\left(\epsilon_{k}^{0}\right)^{2} \sin ^{2} \Delta_{k}, \\
& \mathcal{D}\left(\rho^{\prime}\right)=\beta^{2} \int_{0}^{\pi} \frac{d k}{2 \pi}\left(\epsilon_{k}^{\tau}-\epsilon_{k}^{0} \cos \Delta_{k}\right)^{2}, \\
& \Delta \mathcal{S}_{\text {irr }}=\beta^{2} \int_{0}^{\pi} \frac{d k}{2 \pi}\left[\left(\epsilon_{k}^{\tau}\right)^{2}-2 \epsilon_{k}^{\tau} \epsilon_{k}^{0} \cos \Delta_{k}+\left(\epsilon_{k}^{0}\right)^{2}\right],
\end{aligned}
$$

showing that, to leading order, all quantities scale with the same order in $\beta$. Note also that these expressions do not assume the quench is infinitesimal. Only that the protocol is a sudden quench is assumed. Next, let us specialize to the case of an infinitesimal quench in $g$. That is, we set $g_{\tau}=$ $g_{0}+\delta g, \delta g \ll 1$ and $\gamma_{\tau}=\gamma_{0}$. In this case we get $\sin \Delta_{k} \simeq$ $-\delta g \gamma_{0} \sin k /\left(\epsilon_{k}^{0}\right)^{2}$ so that Eqs. (22a)-(22c) simplify to

$$
\begin{aligned}
& C\left(\rho^{\prime}\right)=\beta^{2} \delta g^{2} \int_{0}^{\pi} \frac{d k}{2 \pi} \gamma_{0}^{2} \frac{\sin ^{2} k}{\left(\epsilon_{k}^{0}\right)^{2}}, \\
& \mathcal{D}\left(\rho^{\prime}\right)=\beta^{2} \delta g^{2} \int_{0}^{\pi} \frac{d k}{2 \pi} \frac{\left(g_{0}-\cos k\right)^{2}}{\left(\epsilon_{k}^{0}\right)^{2}}, \\
& \Delta \mathcal{S}_{\text {irr }}=\frac{1}{2} \beta^{2} \delta g^{2} .
\end{aligned}
$$

From Eq. (23a) it is clear that for this type of quench, the coherence term is maximal for the Ising model $\left(\gamma_{0}=1\right)$, decreasing monotonically with $\gamma_{0}$ until it vanishes in the $X X$ case $\left(\gamma_{0}=0\right)$. In particular, for $\gamma_{0}=1$, the integral in Eq. (23a) may be evaluated analytically, to give

$$
\left.C\left(\rho^{\prime}\right)\right|_{\gamma_{0}=1}= \begin{cases}\frac{\beta^{2} \delta g^{2}}{4} & \text { for }\left|g_{0}\right| \leqslant 1, \\ \frac{\beta^{2} \delta g^{2}}{4 g_{0}^{2}} & \text { for }\left|g_{0}\right|>1 .\end{cases}
$$

This result is quite interesting. First, comparing with Eq. (23c), we see that when $\left|g_{0}\right| \leqslant 1$, half of all the nonequilibrium lag is due to quantum coherence. This is somewhat counterintuitive since this is the high-temperature limit, where 

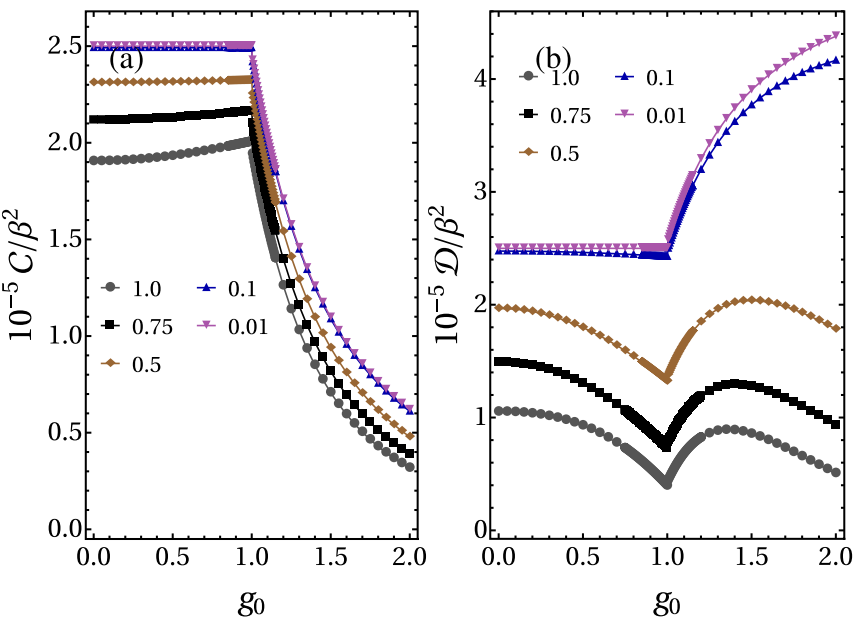

FIG. 2. High-temperature behavior of (a) $\mathcal{C}\left(\rho^{\prime}\right) / \beta^{2}$ and (b) $\mathcal{D}\left(\rho^{\prime}\right) / \beta^{2}$ as functions of $g_{0}$, computed using Eqs. (19) and (21) for quenches in $g$ with amplitude $\left|g_{\tau}-g_{0}\right|=0.01$ and fixed $\gamma_{0}=1$. Different curves correspond to different values of $\beta$. The curves in (a) approach Eq. (24) for high temperatures. Both quantities present a kink at the critical point.

one would expect quantum coherent effects to play a marginal role.

Second, and perhaps even more impressive, we see that Eq. (24) behaves differently in the two phases. And while being continuous, it presents a kink at the critical point. This behavior is plotted in Fig. 2(a). Results for $\mathcal{D}\left(\rho^{\prime}\right) / \beta^{2}$ in the same range of parameters are presented in Fig. 2(b). The high-temperature behavior of the coherence term therefore reflects the nature of the quantum phase transition (which occurs at zero temperature). We are unable to provide an intuitive justification for this behavior. And to the best of our knowledge, we are unaware of any other high-temperature quantities which present nonanalyticities at a quantum critical point. Of course, whether this behavior is experimentally assessable is a complicated question, which has to be addressed in a case-by-case basis. In general $C\left(\rho^{\prime}\right)$ is not directly related to an observable, so that measuring it experimentally will in general be highly nontrivial (requiring full state tomography). However, $\mathcal{D}\left(\rho^{\prime}\right)$ also presents similar signatures and, in principle, is much more easily measurable since it depends only on measurements in the energy basis.

We can similarly perform a quench in the anisotropy parameter, keeping $g_{\tau}=g_{0}$ and setting $\gamma_{\tau}=\gamma_{0}+\delta \gamma$. In this case we get $\sin \Delta_{k} \simeq \delta \gamma\left(g_{0}-\cos k\right) \sin k /\left(\epsilon_{k}^{0}\right)^{2}$. Eqs. (22a)(22c) then simplify to

$$
\begin{aligned}
& C\left(\rho^{\prime}\right)=\beta^{2} \delta \gamma^{2} \int_{0}^{\pi} \frac{d k}{2 \pi} \frac{\left(g_{0}-\cos k\right)^{2} \sin ^{2} k}{\left(\epsilon_{k}^{0}\right)^{2}}, \\
& \mathcal{D}\left(\rho^{\prime}\right)=\beta^{2} \delta \gamma^{2} \int_{0}^{\pi} \frac{d k}{2 \pi} \gamma_{0}^{2} \frac{\sin ^{4} k}{\left(\epsilon_{k}^{0}\right)^{2}}, \\
& \Delta \mathcal{S}_{\text {irr }}=\frac{1}{4} \beta^{2} \delta \gamma^{2} .
\end{aligned}
$$

What is interesting to note in this case is that if we initially have an $X X$ chain, $\gamma_{0}=0$, the population mismatch due to the small quench in the anisotropy parameter vanishes, $\left.D\left(\rho^{\prime}\right)\right|_{\gamma_{0}=0}=0$, and all entropy production is due to coherence, independently of the value of the applied field $g_{0}$.

The above results show that there is an interplay between $C$ and $\mathcal{D}$ for high temperatures, as we go from the $X X$ to the Ising model and as we change from a quench in the field to a quench in the anisotropy. For a quench in the field, the coherence contribution to the entropy production vanishes in an $X X$ chain and increases as we go up to the Ising model, where it reaches a maximum, contributing to half the total production of entropy. For a quench in the anisotropy, in contrast, it is $\mathcal{D}$ that vanishes in a initial $X X$ chain, with all entropy production becoming a consequence of the generation of coherence in the quench protocol. As $\gamma_{0}$ is increased, $C$ steadily decreases, reaching a minimum for the Ising model.

\section{B. Low-temperature limit}

For large $\beta$, Eqs. (19)-(21) can be approximated by

$$
\begin{aligned}
& C\left(\rho^{\prime}\right)=\int_{0}^{\pi} \frac{d k}{2 \pi}\left[-p_{k} \ln p_{k}-\left(1-p_{k}\right) \ln \left(1-p_{k}\right)\right], \\
& \mathcal{D}\left(\rho^{\prime}\right)=4 \beta \int_{0}^{\pi} \frac{d k}{2 \pi} \epsilon_{k}^{\tau} p_{k}-C\left(\rho^{\prime}\right), \\
& \Delta \mathcal{S}_{\text {irr }}=4 \beta \int_{0}^{\pi} \frac{d k}{2 \pi} \epsilon_{k}^{\tau} p_{k},
\end{aligned}
$$

where $p_{k}=\sin ^{2}\left(\Delta_{k} / 2\right)$. Quite interestingly, the integrand in Eq. (26a) is seen to be nothing but the binary Shannon entropy associated with the two-point distribution $\left(p_{k}, 1-p_{k}\right)$ (for each $k$ ). The physical interpretation of $p_{k}$ can be understood from Eq. (16), which shows that $p_{k}=\sin ^{2}\left(\Delta_{k} / 2\right)$ is nothing but the probability of the unoccupied (occupied) prequench modes $\pm k$ to become occupied (unoccupied) after the quench. With this picture in mind, the nonequilibrium lag Eq. (26c) is seen to result solely from this change in occupation, whereas the coherence reflects the entropy associated with this occupation probability.

A notable thing about Eq. (26a), is that it does not depend on $\beta$, unlike $\mathcal{D}\left(\rho^{\prime}\right)$ and $\Delta \mathcal{S}_{\text {irr }}$. This means that, as the temperature is decreased, the relative contribution of $C\left(\rho^{\prime}\right)$ to $\Delta \mathcal{S}_{\text {irr }}$ becomes increasingly less important.

We start our analysis of Eqs. (26a)-(26c) by considering quenches in $g$, with $\gamma_{0}=1$ (Ising). The results are shown in Fig. 3, where we plot $C\left(\rho^{\prime}\right)$ and $\mathcal{D}\left(\rho^{\prime}\right) / \beta$. Clearly, as $\beta \rightarrow$ $\infty$ the latter becomes dominant. As a consequence $\Delta \mathcal{S}_{\text {irr }} \simeq$ $\mathcal{D}\left(\rho^{\prime}\right)$. This is a consequence of the fact that, in this case, changes in the Hamiltonian lead to a significant production of excitations, thus causing the contribution from populations to become dominant. Indeed, in this limit the nonequilibrium lag is known to be proportional to the magnetic susceptibility $\chi=-\partial^{2} F / \partial g_{0}^{2}$ (where $F$ is the equilibrium free energy), according to the relation $[63,102]$

$$
\Delta \mathcal{S}_{\text {irr }}=\beta \delta g^{2} \chi .
$$

As a consequence, $\Delta \mathcal{S}_{\text {irr }} / \beta \delta g^{2}$ diverges logarithmically around the critical points $\left|g_{0}\right|=1[104,105,107]$. This divergence is due solely to the changes in populations.

The coherence in Fig. 3(a), however, does not diverge, which we emphasize by including a plot of $\beta \rightarrow \infty$ in 

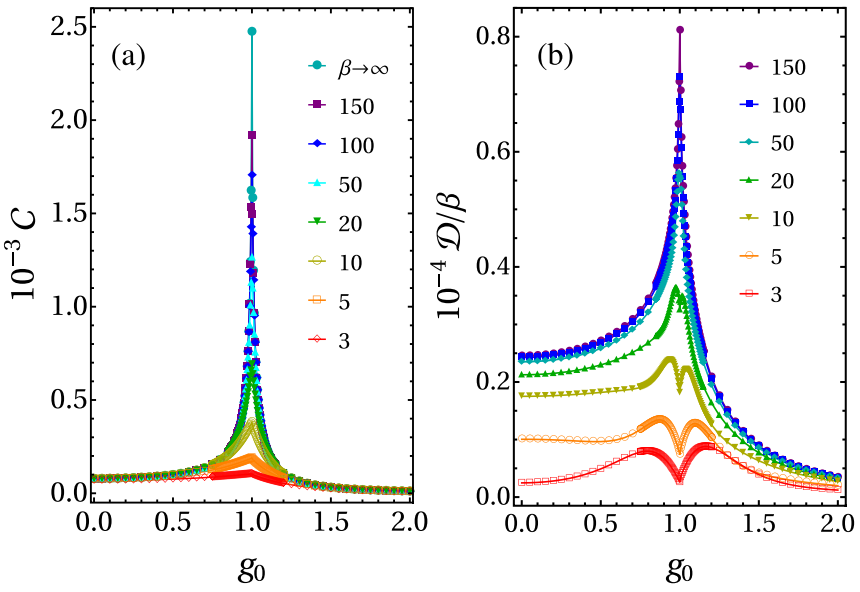

FIG. 3. Low-temperature behavior of (a) $\mathcal{C}\left(\rho^{\prime}\right)$ and (b) $\mathcal{D}\left(\rho^{\prime}\right) / \beta$ for several $\beta$ as functions of $g_{0}$, computed using Eqs. (19) and (21) for quenches in $g$ with amplitude $\left|g_{\tau}-g_{0}\right|=0.01$ and fixed $\gamma_{0}=1$. The curves in (a) approach Eq. (26a) in the limit $T=\frac{1}{\beta} \rightarrow 0$.

Fig. 3(a)-the solid circle curve. Instead, $C\left(\rho^{\prime}\right)$ shows a cusp at the critical point. In fact, Eq. (26a) is bounded from above by $\frac{1}{2} \ln 2$, with this maximum value occurring only for $p_{k}=$ $1 / 2$ for all $k$ 's. From our numerical analysis we also find that the height of the cusp at $g_{0}=1$ approaches the line $\delta g / 4$, at $T=0$. This can be verified analytically by noting that

$$
\begin{aligned}
& C\left(\rho^{\prime}\left[T=0, g_{0}=1\right]\right)=\int_{0}^{2} \frac{d k}{2 \pi} F_{k}+O\left(\delta g^{2} \ln \delta g^{2}\right), \\
& F_{k}=-\frac{1-f_{k}}{2} \ln \frac{1-f_{k}}{2}-\frac{1+f_{k}}{2} \ln \frac{1+f_{k}}{2},
\end{aligned}
$$

and where $f_{k}=k(1+\delta g / 2) / \sqrt{\delta g^{2}+(1+\delta g) k^{2}}$ coincides with $\cos \Delta_{k}\left[g_{0}=1\right]=1-2 p_{k}$ for small $k$. In the interval $2<k<\pi$, we have for the integrand in Eq. (26a), $I_{k}=$ $-p_{k} \ln p_{k}-\left(1-p_{k}\right) \ln \left(1-p_{k}\right)=O\left(\delta g^{2} \ln \delta g^{2}\right)$, when $\delta g \ll$ 1. Moreover, when $0<k<2, I_{k}-F_{k}$ is a monotonically increasing function of $k$, with $F_{k=2}=0$. Finally, the integral of $F_{k} / 2 \pi$ in $0<k<2$ gives $\delta g / 4+O\left(\delta g^{2} \ln \delta g^{2}\right)$. At the critical point, in the limit $\delta g \rightarrow 0, p_{k} \rightarrow 0$ for all $k \neq 0$, while $p_{k=0}=1 / 2$. Furthermore, for the derivative of $C\left(\rho^{\prime}\right)$ with
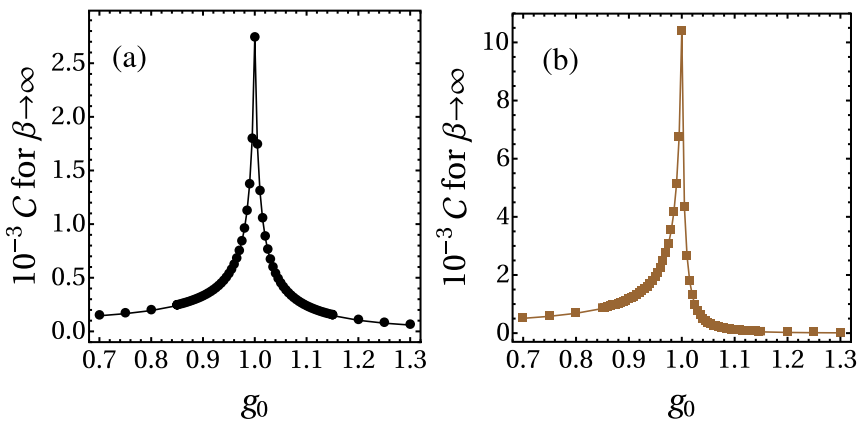

FIG. 4. $C\left(\rho^{\prime}\right)$ vs. $g_{0}$ for $T=0$ and quenches in $g$ of magnitude $\left|g_{\tau}-g_{0}\right|=0.01$. (a) Close to the Ising case, $\gamma_{0}=0.9$ and (b) close to the $X X$ case, $\gamma=0.2$. The curves show how the cusp of $C\left(\rho^{\prime}\right)$ becomes more asymmetric as $\gamma_{0}$ is reduced.
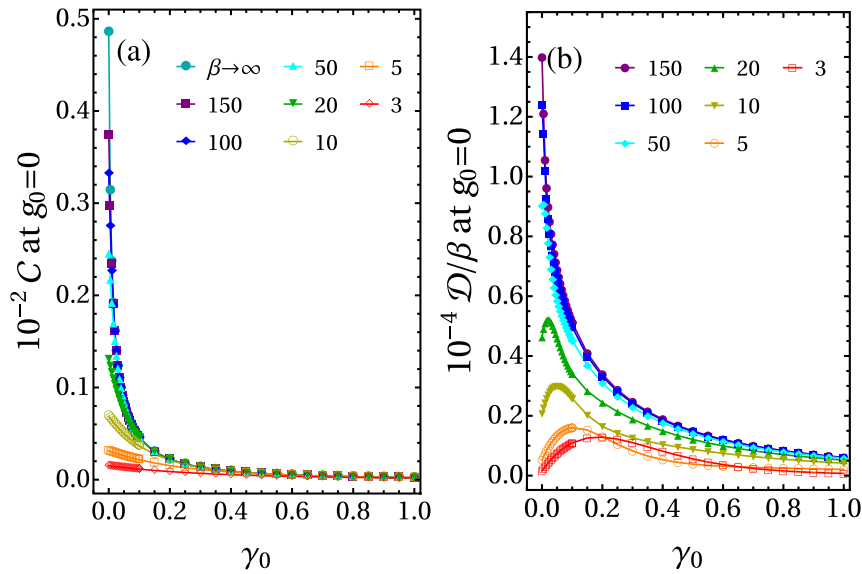

FIG. 5. Low-temperature behavior of (a) $\mathcal{C}\left(\rho^{\prime}\right)$ and (b) $\mathcal{D}\left(\rho^{\prime}\right) / \beta$ for several $\beta$ as functions of $\gamma_{0}$, computed using Eqs. (19) and (21) for quenches in $\gamma$ with amplitude $\left|\gamma_{\tau}-\gamma_{0}\right|=0.01$ and fixed $g_{0}=0$.

respect to $g_{0}\left(-g_{\tau}\right)$ we find that, when $\delta g \rightarrow 0$, it approaches $1 / 4$ at $g_{0}\left(g_{\tau}\right)=1$, while it goes to zero elsewhere.

The shape of the cusp in $C\left(\rho^{\prime}\right)$ depends on the value of $\gamma_{0}$. This is presented in Fig. 4, where we plot $C\left(\rho^{\prime}\right)$ for $\beta \rightarrow \infty$ for different values of $\gamma_{0}$. As can be seen, it changes from a very symmetric form for larger values of $\gamma_{0}$ to an increasingly asymmetric format as $\gamma_{0}$ decreases.

We also studied the case of quenches in the anisotropy, with fixed field. In this context, the coherence decreases with increasing $\gamma_{0}$ and has its maximal value for a vanishing field, see Fig. 5.

\section{Ratio $C\left(\rho^{\prime}\right) / \Delta \mathcal{S}_{\text {irr }}$}

Next we combine the high- and low-temperature results and perform an analysis of the relative contribution $C\left(\rho^{\prime}\right) / \Delta \mathcal{S}_{\text {irr. }}$. Results for quenches in $g$, with $\gamma_{0}=1$ and several values of $\beta$ are shown in Fig. 6. In the case of high temperatures, e.g., $\beta=0.1-$ empty circle curve in the center,

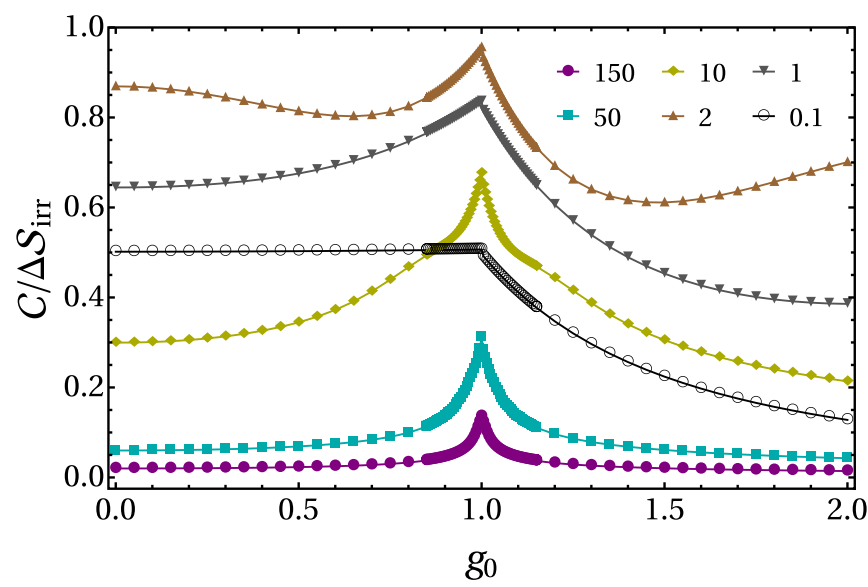

FIG. 6. Ratio $C / \Delta S_{\text {irr }}$ vs. $g_{0}$ for several $\beta$ and quenches in $g$ with magnitude $\left|g_{\tau}-g_{0}\right|=0.01$, with fixed $\gamma_{0}=1$. For $\beta=0.1$ the ratio approaches the limit in Eq. (24). A notable feature is the presence of an optimal temperature $\beta \approx 2$, in which almost all entropy production is due to the generation of coherence. 


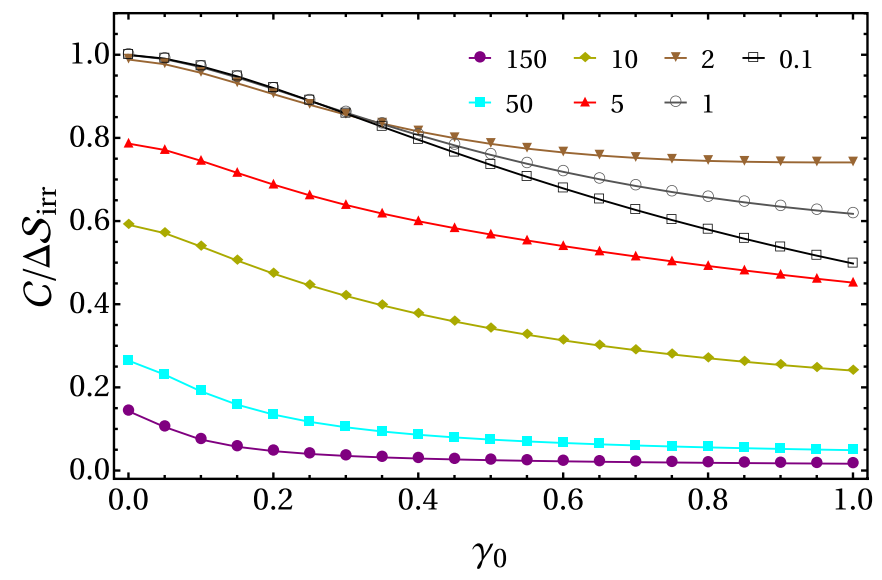

FIG. 7. $\mathcal{C}\left(\rho^{\prime}\right) / \Delta \mathcal{S}_{\text {irr }}$ as a function of $\gamma_{0}$ for a series of quenches in the anisotropy parameter with amplitude $\left|\gamma_{\tau}-\gamma_{0}\right|=0.01$, for several $\beta$, at $g_{0}=1$. It shows that, for small $\beta$ and $\gamma_{0}=0$, all entropy production is due to the generation of coherence for this type of quench.

this fraction approaches $1 / 2$, which is the limit predicted by Eq. (24). Similarly, for low temperatures, the ratio tends to zero, as discussed in Sec. III B. The notable features of Fig. 6, however, is for intermediate temperatures, where the results are not at all intuitive. First, there exists an "optimal" temperature, around $\beta \approx 2$, for which the ratio approaches unity, so that almost all entropy produced stems from coherence. This happens because as the dependence of $\mathcal{D}\left(\rho^{\prime}\right)$ on $\beta$ changes from $\beta^{2}$, in the high-temperature limit, to a linear dependence on $\beta$ in the low-temperature limit, there is a range of temperatures in which the coherence generation for a given quench increases more rapidly than the population imbalance. Second, for large $\beta$, even though the ratio is generally small, there is nonetheless a substantial increase in the vicinity of the critical point. This is a consequence of the sharp peak in the coherence in this region, as shown in Fig. 3. However, since the coherence saturates for increasing $\beta$ while the entropy production always increases, this peak in the fraction $C / \Delta \mathcal{S}_{\text {irr }}$ approaches zero as the temperature tends to zero.

A similar analysis for quenches in the anisotropy parameter is shown in Fig. 7. The curve for $\beta=0.1$ (empty square line) show how the ratio approaches unity as $\gamma_{0} \rightarrow 0$, as previously discussed in Sec. III A. Notably, for intermediate values of $\beta$, between $\beta=5$ (up triangle curve) to $\beta=10$ (diamond curve), in the critical point, the coherence accounts for a large part of the production of entropy, between $25 \%$ and $80 \%$, for any value of the initial anisotropy. Again for large $\beta$, this ratio approaches zero.

\section{CONCLUSION}

We investigated the genuinely quantum-mechanical contribution of the generation of coherence to the production of entropy for quenches in the transverse field and in the anisotropy parameter of an $X Y$ model. We showed that the generation of coherence is intimately related to the rotation in the basis that diagonalizes the system's Hamiltonian when the quench protocol is performed.

For large temperatures (small $\beta=1 / T$ ), we showed that there is an interplay between the coherent and incoherent contributions. For small quenches in the transverse field, the coherence increases steadily with the anisotropy parameter, reaching a maximum for the Ising model. For small quenches in the anisotropy, instead, we found that the coherence is the sole responsible for the entropy production if the systems starts in an $X X$ chain. As the initial anisotropy is increased, the coherence decreases and reaches a minimum in the Ising model. Moreover, we showed that even in this limit of temperatures the nature of the quantum phase transition of the system (which occurs at $T=0$ ) is reflected in the coherence, which presents a kink at the critical point.

For small temperatures, we found a saturation in the contribution from coherence. This results from the fact that in such cases any change in the Hamiltonian leads to excitations on the system, which forcibly makes the production of entropy to be associated with the changes in population on the system. We also showed that the behavior of the coherence around the critical point, for quenches in the field, does not present a discontinuity, but rather a cusp. Notwithstanding, the entropy production still diverges, which is solely due to the changes in populations.

Concerning the generality of our results, they are based upon the assumptions of an initial thermal state that is instantaneously quenched, and on that the eigenbases of the initial and final Hamiltonians are related by a Bogoliubov transformation, i.e., Eq. (14), and we expect them to hold whenever such assumptions can be made for other systems.

Finally, we analyzed the relative contribution of coherence to the total entropy production. For quenches in the transverse field in the Ising model, we showed that for small $\beta$ this fraction approaches $1 / 2$ in the ferromagnetic region. We also found that at certain temperatures the coherence can account for almost all the entropy production. For quenches in the anisotropy, the ratio of coherence to the production of entropy remains large even for intermediate $\beta$, for any initial anisotropy.

\section{ACKNOWLEDGMENTS}

The authors acknowledge fruitful discussions with $\mathrm{M}$. Perarnau-Llobet, M. Scandi, D. Uip, S. Campbell, L. H. Mandetta, and J. Goold. We acknowledge financial support from the Brazilian agencies Conselho Nacional de Desenvolvimento Científico e Tecnológico and Coordenação de Aperfeiçoamento de Pessoal de Nível Superior. G.T.L. and A.P.V. acknowledge the São Paulo Research Foundation FAPESP (Grants No. 2018/12813-0, No. 2017/50304-7, No. 2017/07973-5, No. 2017/07248-9).
[1] C. Jarzynski, Phys. Rev. Lett. 78, 2690 (1997).

[2] J. Kurchan, J. Phys. A: Math. Gen. 31, 3719 (1998).
[3] P. Talkner, E. Lutz, and P. Hänggi, Phys. Rev. E 75, 050102(R) (2007). 
[4] R. Kawai, J. M. R. Parrondo, and C. Van den Broeck, Phys. Rev. Lett. 98, 080602 (2007).

[5] S. Vaikuntanathan and C. Jarzynski, Europhys. Lett. 87, 60005 (2009).

[6] J. M. Parrondo, C. Van Den Broeck, and R. Kawai, New J. Phys. 11, 073008 (2009).

[7] S. Deffner and E. Lutz, Phys. Rev. Lett. 105, 170402 (2010).

[8] T. B. Batalhão, A. M. Souza, R. S. Sarthour, I. S. Oliveira, M. Paternostro, E. Lutz, and R. M. Serra, Phys. Rev. Lett. 115, 190601 (2015).

[9] E. Fermi, Thermodynamics (Dover Publications, Mineola, NY, 1956), p. 160.

[10] H. Spohn and J. L. Lebowitz, Adv. Chem. Phys. 38, 109 (1978).

[11] H. P. Breuer, Phys. Rev. A 68, 032105 (2003).

[12] J. P. Santos, L. C. Céleri, G. T. Landi, and M. Paternostro, Nat. Quantum Info. 5, 23 (2019).

[13] Note that the entropy production is not the change in entropy of the system, which according to Clausius' principle, reads $\Delta S=\Delta S_{\text {irr }}+Q / T$, where $Q$ is the heat that flows from the system to the bath. The entropy production is therefore only part of $\Delta S$ (a part which is always nonnegative).

[14] C. Jarzynski, Phys. Rev. E 56, 5018 (1997).

[15] B. Derrida and J. L. Lebowitz, Phys. Rev. Lett. 80, 209 (1998).

[16] G. E. Crooks, J. Stat. Phys. 90, 1481 (1998).

[17] J. Lebowitz and H. Spohn, J. Stat. Phys. 95, 333 (1999).

[18] C. Jarzynski, J. Stat. Phys. 96, 415 (1999).

[19] C. Maes, J. Stat. Phys. 95, 367 (1999).

[20] C. Jarzynski, Proc. Nat. Acad. Sci. U.S.A. 98, 3636 (2001).

[21] G. E. Crooks, Phys. Rev. E 61, 2361 (2000).

[22] C. Jarzynski, J. Stat. Phys. 98, 77 (2000).

[23] S. Mukamel, Phys. Rev. Lett. 90, 170604 (2003).

[24] D. Andrieux and P. Gaspard, J. Chem. Phys. 121, 6167 (2004).

[25] T. Monnai, Phys. Rev. E 72, 027102 (2005).

[26] J. Teifel and G. Mahler, Phys. Rev. E 76, 051126 (2007).

[27] G. E. Crooks, J. Stat. Mech.: Theory Exp. (2008) P10023.

[28] M. F. Gelin and D. S. Kosov, Phys. Rev. E 78, 011116 (2008).

[29] C. Jarzynski, European Phys. J. B 64, 331 (2008).

[30] P. Talkner, M. Campisi, and P. Hänggi, J. Stat. Mech.: Theory Exp. (2009) P02025.

[31] J. Teifel and G. Mahler, Phys. Rev. E 83, 041131 (2011).

[32] L. Mazzola, G. De Chiara, and M. Paternostro, Phys. Rev. Lett. 110, 230602 (2013).

[33] R. Dorner, S. R. Clark, L. Heaney, R. Fazio, J. Goold, and V. Vedral, Phys. Rev. Lett. 110, 230601 (2013).

[34] P. Talkner, M. Morillo, J. Yi, and P. Hänggi, New J. Phys. 15, 095001 (2013).

[35] D. A. Sivak and G. E. Crooks, Phys. Rev. Lett. 108, 150601 (2012).

[36] J. Hoppenau and A. Engel, J. Stat. Mech.: Theory Exp. (2013) P06004.

[37] G. Watanabe, B. P. Venkatesh, P. Talkner, M. Campisi, and P. Hänggi, Phys. Rev. E 89, 032114 (2014).

[38] A. J. Roncaglia, F. Cerisola, and J. P. Paz, Phys. Rev. Lett. 113, 250601 (2014).

[39] F. Plastina, A. Alecce, T. J. G. Apollaro, G. Falcone, G. Francica, F. Galve, N. Lo Gullo, and R. Zambrini, Phys. Rev. Lett. 113, 260601 (2014).
[40] P. Skrzypczyk, A. J. Short, and S. Popescu, Nat. Commun. 5, 4185 (2014)

[41] N. Y. Halpern, A. J. Garner, O. C. Dahlsten, and V. Vedral, New J. Phys. 17, 095003 (2015).

[42] P. Solinas and S. Gasparinetti, Phys. Rev. E 92, 042150 (2015).

[43] K. Funo, Y. Murashita, and M. Ueda, New J. Phys. 17, 075005 (2015).

[44] Á. M. Alhambra, L. Masanes, J. Oppenheim, and C. Perry, Phys. Rev. X 6, 041017 (2016).

[45] Á. M. Alhambra, J. Oppenheim, and C. Perry, Phys. Rev. X 6, 041016 (2016).

[46] P. Talkner and P. Hänggi, Phys. Rev. E 93, 022131 (2016).

[47] F. Jin, R. Steinigeweg, H. De Raedt, K. Michielsen, M. Campisi, and J. Gemmer, Phys. Rev. E 94, 012125 (2016).

[48] A. Chenu, I. L. Egusquiza, J. Molina-Vilaplana, and A. del Campo, Sci. Rep. 8, 12634 (2018).

[49] P. Solinas, H. J. D. Miller, and J. Anders, Phys. Rev. A 96, 052115 (2017).

[50] A. Bartolotta and S. Deffner, Phys. Rev. X 8, 011033 (2018).

[51] J. J. Park, S. W. Kim, and V. Vedral (2017), arXiv:1705.01750.

[52] M. Perarnau-Llobet, E. Bäumer, K. V. Hovhannisyan, M. Huber, and A. Acin, Phys. Rev. Lett. 118, 070601 (2017).

[53] R. Sampaio, S. Suomela, T. Ala-Nissila, J. Anders, and T. G. Philbin, Phys. Rev. A 97, 012131 (2018).

[54] C. Elouard, D. A. Herrera-Martí, M. Clusel, and A. Auffèves, npj Quant. Info. 3, 9 (2017).

[55] P. G. Di Stefano, J. J. Alonso, E. Lutz, G. Falci, and M. Paternostro, Phys. Rev. B 98, 144514 (2018).

[56] B. B. Wei and M. B. Plenio, New J. Phys. 19, 023002 (2017).

[57] C. Elouard, D. Herrera-Martí, B. Huard, and A. Auffèves, Phys. Rev. Lett. 118, 260603 (2017).

[58] M. Lostaglio, Phys. Rev. Lett. 120, 040602 (2018).

[59] G. Guarnieri, N. H. Y. Ng, K. Modi, J. Eisert, M. Paternostro, and J. Goold, Phys. Rev. E 99, 050101(R) (2019).

[60] G. Manzano, J. M. Horowitz, and J. M. R. Parrondo, Phys. Rev. X 8, 031037 (2018).

[61] G. Francica, J. Goold, and F. Plastina, Phys. Rev. E 99, 042105 (2019).

[62] G. De Chiara, P. Solinas, F. Cerisola, and A. J. Roncaglia, in Thermodynamics in the Quantum Regime-Recent Progress and Outlook, edited by F. Binder, L. A. Correa, C. Gogolin, J. Anders, and G. Adesso (Springer International Publishing, Berlin, 2019), pp. 337-362.

[63] L. Fusco, S. Pigeon, T. J. G. Apollaro, A. Xuereb, L. Mazzola, M. Campisi, A. Ferraro, M. Paternostro, and G. De Chiara, Phys. Rev. X 4, 031029 (2014).

[64] G. Francica, J. Goold, M. Paternostro, and F. Plastina, Nature Quant. Info. 3, 12 (2017).

[65] M. Zhong and P. Tong, Phys. Rev. E 91, 032137 (2015).

[66] T. J. G. Apollaro, G. Francica, M. Paternostro, and M. Campisi, Phys. Scr. 2015, 014023 (2015).

[67] A. del Campo, J. Goold, and M. Paternostro, Sci. Rep. 4, 6208 (2014).

[68] M. Brunelli, A. Xuereb, A. Ferraro, G. De Chiara, N. Kiesel, and M. Paternostro, New J. Phys. 17, 035016 (2015).

[69] A. E. Allahverdyan and T. M. Nieuwenhuizen, Phys. Rev. E 71, 066102 (2005). 
[70] G. E. Crooks and C. Jarzynski, Phys. Rev. E 75, 021116 (2007).

[71] P. Talkner, P. S. Burada, and P. Hänggi, Phys. Rev. E 78, 011115 (2008).

[72] S. Deffner and E. Lutz, Phys. Rev. E 77, 021128 (2008).

[73] S. Dorosz, T. Platini, and D. Karevski, Phys. Rev. E 77, 051120 (2008).

[74] R. Dorner, J. Goold, C. Cormick, M. Paternostro, and V. Vedral, Phys. Rev. Lett. 109, 160601 (2012).

[75] A. Ryabov, M. Dierl, P. Chvosta, M. Einax, and P. Maass, J. Phys. A: Math. Theor. 46, 075002 (2013).

[76] M. Paternostro, G. De Chiara, A. Ferraro, M. Campisi, J. Goold, F. L. Semião, F. Plastina, and V. Vedral, J. Stat. Mech.: Theory Exp. (2019) 104014.

[77] M. Perarnau-Llobet, K. V. Hovhannisyan, M. Huber, P. Skrzypczyk, N. Brunner, and A. Acín, Phys. Rev. X 5, 041011 (2015).

[78] A. Sindona, J. Goold, N. Lo Gullo, and F. Plastina, New J. Phys. 16, 045013 (2014).

[79] G. D. Chiara, A. J. Roncaglia, and J. P. Paz, New J. Phys. 17, 035004 (2015).

[80] E. G. Arrais, D. A. Wisniacki, L. C. Céleri, N. G. de Almeida, A. J. Roncaglia, and F. Toscano, Phys. Rev. E 98, 012106 (2018).

[81] M. Łobejko, J. Łuczka, and P. Talkner, Phys. Rev. E 95, 052137 (2017).

[82] F. A. Bayocboc and F. N. C. Paraan, Phys. Rev. E 92, 032142 (2015).

[83] A. Silva, Phys. Rev. Lett. 101, 120603 (2008).

[84] M. Heyl, A. Polkovnikov, and S. Kehrein, Phys. Rev. Lett. 110, 135704 (2013).

[85] Z. Fei and H. T. Quan, Phys. Rev. Res. 1, 033175 (2019).

[86] Z. Fei, N. Freitas, V. Cavina, H. T. Quan, and M. Esposito, Phys. Rev. Lett. 124, 170603 (2020).

[87] J. Liphardt, S. Dumont, S. B. Smith, I. Tinoco, and C. Bustamante, Science 296, 1832 (2002).

[88] F. Douarche, S. Ciliberto, a. Petrosyan, and I. Rabbiosi, Europhys. Lett. 70, 593 (2005).

[89] D. Collin, F. Ritort, C. Jarzynski, S. B. Smith, I. Tinoco, and C. Bustamante, Nature 437, 231 (2005).

[90] T. Speck, V. Blickle, C. Bechinger, and U. Seifert, Europhys. Lett. 79, 30002 (2007).

[91] O. P. Saira, Y. Yoon, T. Tanttu, M. Möttönen, D. V. Averin, and J. P. Pekola, Phys. Rev. Lett. 109, 180601 (2012).

[92] J. V. Koski, T. Sagawa, O. P. Saira, Y. Yoon, A. Kutvonen, P. Solinas, M. Möttönen, T. Ala-Nissila, and J. P. Pekola, Nat. Phys. 9, 644 (2013).

[93] T. B. Batalhão, A. M. Souza, L. Mazzola, R. Auccaise, R. S. Sarthour, I. S. Oliveira, J. Goold, G. De Chiara, M. Paternostro, and R. M. Serra, Phys. Rev. Lett. 113, 140601 (2014).
[94] S. An, J.-N. Zhang, M. Um, D. Lv, Y. Lu, J. Zhang, Z.-Q. Yin, H. T. Quan, and K. Kim, Nat. Phys. 11, 193 (2014).

[95] M. A. A. Talarico, P. B. Monteiro, E. C. Mattei, E. I. Duzzioni, P. H. Souto Ribeiro, and L. C. Céleri, Phys. Rev. A 94, 042305 (2016).

[96] Z. Zhang, T. Wang, L. Xiang, Z. Jia, P. Duan, W. Cai, Z. Zhan, Z. Zong, J. Wu, L. Sun, Y. Yin, and G. Guo, New J. Phys. 20, 085001 (2018).

[97] A. Smith, Y. Lu, S. An, X. Zhang, J.-N. Zhang, Z. Gong, H. T. Quan, C. Jarzynski, and K. Kim, New J. Phys. 20, 013008 (2018).

[98] W. H. Zurek, Phys. Rev. D 24, 1516 (1981).

[99] M. Lostaglio, D. Jennings, and T. Rudolph, Nat. Commun. 6, 6383 (2015).

[100] H. J. D. Miller, M. Scandi, J. Anders, and M. Perarnau-Llobet, Phys. Rev. Lett. 123, 230603 (2019).

[101] M. Scandi, H. J. D. Miller, J. Anders, and M. Perarnau-Llobet, Phys. Rev. Research 2, 023377 (2020).

[102] A. Gambassi and A. Silva, Statistics of the work in quantum quenches, universality and the critical Casimir effect (2011), arXiv:1106.2671.

[103] J. Goold, F. Plastina, A. Gambassi, and A. Silva, in Thermodynamics in the Quantum Regime-Recent Progress and Outlook, edited by F. Binder, L. A. Correa, G. C, J. Anders, and G. Adesso (Springer, Berlin, 2018), pp. 317-336.

[104] E. Mascarenhas, H. Bragança, R. Dorner, M. França Santos, V. Vedral, K. Modi, and J. Goold, Phys. Rev. E 89, 062103 (2014).

[105] S. Sharma and A. Dutta, Phys. Rev. E 92, 022108 (2015).

[106] F. Cosco, M. Borrelli, P. Silvi, S. Maniscalco, and G. De Chiara, Phys. Rev. A 95, 063615 (2017).

[107] S. Paganelli and T. J. G. Apollaro, Int. J. Mod. Phys.s B 31, 1750065 (2017).

[108] Q. Wang, D. Cao, and H. T. Quan, Phys. Rev. E 98, 022107 (2018).

[109] A. Bayat, T. J. G. Apollaro, S. Paganelli, G. De Chiara, H. Johannesson, S. Bose, and P. Sodano, Phys. Rev. B 93, 201106(R) (2016).

[110] A. Pelissetto, D. Rossini, and E. Vicari, Phys. Rev. E 97, 052148 (2018).

[111] D. Nigro, D. Rossini, and E. Vicari, J. Stat. Mech.: Theory Exp. (2019) 023104.

[112] E. Vicari, Phys. Rev. A 99, 043603 (2019).

[113] L. Villa and G. De Chiara, Quantum 2, 42 (2018).

[114] E. H. Lieb, T. Schultz, and D. Mattis, Ann. Phys. 16, 407 (1961).

[115] S. Sachdev, Quantum Phase Transitions (Cambridge University Press, Cambridge, UK, 2011).

[116] B. Damski and M. M. Rams, J. Phys. A: Math. Theor. 47, 025303 (2014). 